\title{
Erector spinae plane block vs. Nonsteroidal anti-inflammatory drugs for severe renal colic pain: a pilot clinical feasibility study
}

\author{
Muhammed Enes Aydin ${ }^{1}$, Erdal Tekin ${ }^{1}$, Elif Oral Ahiskalioglu ${ }^{1}$, Irem Ates ${ }^{1}$, Selahattin \\ Karagoz $^{1}$, Omerul Faruk Aydin ${ }^{2}$, Fatih Ozkaya ${ }^{1}$, and Ali Ahiskalioglu ${ }^{1}$ \\ ${ }^{1}$ Ataturk University \\ ${ }^{2}$ Yeni Yuzyil Universitesi
}

August 12, 2020

\begin{abstract}
Aim: Ultrasound-guided plane blocks are increasingly used in the multi-modal analgesic concept for reducing opioid consumption. The present study was conducted to compare the analgesic effect of intravenous nonsteroidal anti-inflammatory drugs (NSAIDs) and erector spinae plane (ESP) block in renal colic patients. Methods: In this prospective randomized study, 40 patients with renal colic pain were randomly assigned to into two groups; Group NSAID ( $\mathrm{n}=20)$ received an intravenous infusion of $50 \mathrm{mg}$ of dexketoprofen trometamol, Group ESP $(\mathrm{n}=20)$ received ultrasound-guided erector spinae plane block with $30 \mathrm{ml}$ $0.25 \%$ bupivacaine at the T8 level. The pain severity of patients was assessed using the visual analog scale (VAS) at baseline, 5., 15., 30., 45. and 60. minutes after intervention. Opioid consumption, patient satisfaction and side effects were recorded. Results: In the ESP group, the VAS scores were significantly lower than the NSAID group at 5., 15., 30., 45. and 60. minutes after the procedure $(\mathrm{P}<0.001)$. Opioid consumption was significantly higher in the NSAID group compared with the ESP group $(10 / 20$ vs. $0 / 20$, respectively; $p<0.001)$. Patient satisfaction was significantly higher in the ESP group $(\mathrm{p}<0.001)$. Conclusions: ESP block can be an alternative, efficient, and safe method for the relief of acute renal colic pain.
\end{abstract}

\section{a. What is already known about the topic:}

Non-steroidal anti-inflammatory drugs, systemic opioid analgesics, trigger point injections and other regional anesthetic methods were commonly used analgesic methods for renal colic pain. The effect of interfacial plane blocks is still limited.

\section{b. What new information this study adds:}

This is the first randomized controlled study to evaluate the use of erector spinae plane block for severe renal colic pain. Erector spinae plane block provides effective analgesia with opioid sparing effects for this indication.

Keywords: Renal colic pain, erector spinae plane block, ultrasound, emergency department

\section{INTRODUCTION}

Pain, which is defined as a rather uncomfortable physical sensation caused due to any illness or injury, is one of the most common causes of consulting with the emergency department (ED). Acute renal colic is a clinical condition characterized by severe, widespread flank pain and often accompanied by urinary tract stones. Its prevalence worldwide is $5-15 \%{ }^{1}$. Laboratory examinations and imaging procedures are performed to expose urolithiasis or to exclude other possible early diagnoses following the first symptomatic treatment like hydration. Those patients for whom emergency surgery is not planned following the diagnosis are generally followed up with analgesic protocols. 
To determine the suitable analgesic protocol, such parameters as medication efficacy, safety and accessibility are determinants along with the patient's pain score. Non-steroidal anti-inflammatory drugs (NSAIDs) and opioids constitute the foundation of today's acute renal colic analgesic protocols. Since opioids can be titrated based on the severity of the pain and has high level of efficacy, they are still commonly used at several healthcare centers. However, due to the risk of drug abuse and respiratory depression, as well as considering its adverse effects such as nausea-vomiting, NSAIDs are accepted as the first line medication since they have an analgesic efficacy similar to that of opioids ${ }^{2}$. Just like opioids, NSAIDs are not innocent either in terms of their adverse effects. Additionally, various agents such as paracetamol, ketamine, calcium channel blockers, alpha blockers, vasopressin analogs and magnesium sulfate were used for analgesic management of acute renal colic ${ }^{3-5}$. In addition to these medical treatments, such regional analgesic techniques as trigger point injection ${ }^{6}$, twelfth intercostal nerve block ${ }^{7}$, and subcutaneous paravertebral block ${ }^{8}$ were also employed. Ultrasound-guided application of regional anesthesia techniques has higher rates of success and lower risk of complications. Especially the plane blocks for which a new form is defined each passing day are way easier to learn and to practice, which makes them preferable by various clinicians.

Moreover, when performed with ultrasound guidance, regional anesthesia techniques have gained a tremendous amount of popularity in eliminating acute pain over the recent years. To conclude, the ultrasound-guided plane blocks have been included in the multi-model analgesic concept as a non-opioid analgesic option ${ }^{9}$. The ultrasound-guided erector spinae plane (ESP) block, which was first defined with thoracic neuropathic pain management by Forero ${ }^{10}$, is currently routinely used in several surgical procedures for postoperative analgesia ${ }^{11,12}$, and it is also used in the analgesic management of various acute and chronic pain ${ }^{13,14}$. We have shared a presentation of three cases to point out to the analgesic efficacy that the ESP block might potentially have in acute renal colic ${ }^{15}$. To the best of our knowledge, the present study is the first randomized pilot clinical study that shows the efficacy of ESP block in the analgesic management of acute renal colic.

The primary aim of the present study is to evaluate the effect of ESP block on the opioid consumption in the analgesic management of acute renal colic while the second one is to evaluate its effect on pain scores.

\section{METHODS}

Including patients aged between 18-65 years that consulted with the ED with complaints of flank pain due to renal colic, the present study is a prospective randomized clinical study. The study was conducted between January 1, 2019 - January 1, 2020. The study was approved by the university's ethics committee. The study is registered at clinicaltrials.gov (NCT04209439). Written informed consent was obtained from all of the study patients. The study was conducted in accordance with the CONSORT guideline and the Declaration of Helsinki.

This pilot clinical feasibility study was conducted under the definitions and protocols described in a published tutorial in a pilot study ${ }^{16}$, and due to lack of previous studies, a convenience sample size of 20 was used for each group.

\section{Study setting and population}

The study was conducted at a single healthcare center, which was the ED of a tertiary university hospital. Excluded patients included from the study those who were planned to be operated urgently, had severe systemic diseases (renal, hepatic, pulmonary, and endocrine diseases), had bilateral obstruction, with a single kidney, anuric patients, pregnant and breastfeeding, allergic to the medication to be used.

After admission to ED, computerized tomography and laboratory procedures were performed for the diagnosis of urolithiasis. Intravenous hydration was administered to all patients as an initial treatment, and the patients were assigned into two equal groups according to computer randomization program as;

Group NSAID: Patients included in the NSAID group were administered a 5 min i.v. injection of $50 \mathrm{mg}$ dexketoprofen trometamol (Arveles $50 \mathrm{mg} / 2 \mathrm{ml}$ ampule, Ufsa Medication Industry and Trade Inc., Turkey) diluted in $20 \mathrm{~mL}$ of normal saline. 
Group ESP: The patient was placed in the seated position. The skin was prepared with antiseptic solutions. Then the transverse process and the erector spina muscle were identified in the sagittal plane with multifrequency linear probe (Toshiba Aplio XG Diagnostic Ultrasound System, 04/2016 Japan) placed longitudinal parasagittal orientation at $3 \mathrm{~cm}$ lateral of the midline at $\mathrm{T} 8$ vertebrae level. While the needle (Braun Stimuplex@ Ultra 360, Melsungen, Germany) was advanced by in-plane technique, plane was verified by performed hydro-dissection with $3 \mathrm{ml}$ saline. ESP block was administered by injection of $30 \mathrm{ml} 0.25 \%$ bupivacaine and into the fascial plane between the transverse process and erector spinae muscle (Figure 1).

\section{Study outcomes}

The two groups of patients included in the study were compared in terms of age, gender, symptoms and findings, localization and size of the stone, vital parameters (electrocardiogram, non-invasive blood pressure, rate of respiration, and oxygen saturation), drug adverse effects and complications.

The pain scores of patients at the admittance were measured using Visual Analog Scale (VAS). VAS is a scale of values between $0-100 \mathrm{~mm}$. The patients were informed that $0 \mathrm{~mm}$ indicates no pain while the most severe pain is indicated in $100 \mathrm{~mm}$. The patients were given the VAS scale to mark the corresponding score on the scale in each measurement. The VAS scores at the admittance and the VAS scores 5., 15., 30., 45. and 60. minutes were recorded. In cases with a VAS score of [?]40 in both groups after post-medication or block procedure, intravenous administration of $1 \mathrm{mcg} / \mathrm{kg}$ of fentanyl (Talinat $0.5 \mathrm{mg} / 10 \mathrm{ml}$ ampule, Vem Medication, Turkey) was determined as the opioid analgesics. The patients whose VAS scores could not be reduced below 40 despite the opioid analgesia were hospitalized at the urology clinic for treatment. The patients were followed up urologically after procedures to prevent hydronephrosis. Patient satisfaction was assessed based on a scale of 1 to 5 (measured as a score of 1 to 5 as follows: very poorly, poorly, moderate, good, and excellent, respectively).

\section{Statistical Analyses}

Statistical analysis was performed using the SPSS software version 20.0 (IBM Corp., Armonk, New York, USA). Distribution of variables was evaluated for normality using the Kolmogorov-Smirnov and histogram tests. Descriptive data were expressed as mean +- standard deviation. Categorical variables were analyzed using the Chi-square test. Normally distributed data comprising continuous variables were analyzed using the Student's $t$-test. The Mann-Whitney U test was used to analyze non-normally distributed data. $\mathrm{p}$ $<0.05$ was considered to be statistically significant.

\section{RESULTS}

The study included 51 patients. 11 patients were excluded due to varying reasons. Each of the two groups included 20 patients (Figure 2).

The mean age of the patients was $38.88+-12.67$ years and $62.5 \%(\mathrm{n}=25)$ were male. The mean ages of the two groups were $35.95+-12.36$ and $41.80+-12.60$, respectively. The vital parameters of the patients at the admittance did not differ at a statistically significant between the groups $(\mathrm{p}>0.05)$. Other demographics and baseline characteristics of the patients are presented (Table 1).

Pain scores of the patients are shown in Table 2. The VAS of the patients at the admittance was 96.25+-6.15 for group NSAID and $98.60+-3.03$ for group ESP. There was not a statistically significant difference between the groups $(\mathrm{p}=0.429)$. VAS scores were significantly lower in the ESP group compared to the NSAID group at 5., 15., 30., 45. and 60. minutes $(\mathrm{p}<0.001)$.

The need for opioid analgesics was significantly lower in Group ESP when compared to the group NSAID $(0 / 20$ versus $10 / 20$, respectively, $\mathrm{p}<0.001)$. Patient satisfaction was significantly higher in the ESP group than NSAID group $(\mathrm{p}<0.001)$ (Table 3$)$. No adverse event occurred in any of the patients in the study.

\section{DISCUSSION}

The mechanism of pain in acute renal colic involves changes in intraluminal pressure, renal blood flow, and 
glomerular filtration rate caused by increased NO and prostaglandin release as a result of urinary system obstruction by stones ${ }^{17}$. The sympathetic preganglionic nerves that form the renal innervation originate from the spinal segments T8 and L1. The ureter has an abundant source of autonomic nerves originating from the celiac, aortorenal, and mesenteric ganglions in addition to superior and inferior hypogastric (pelvic) plexus. The sympathetic response to the ureter arises from the preganglionic fibers of the lumbar segments T11, T12, and L1. Distention of the renal capsule and the collecting system causes stimulation of renal pain fibers that carry signals through the sympathetic nerves, thus resulting in the visceral-type referred pain in the flank, groin, or scrotal (labial) regions ${ }^{18}$.

The time to reach effective analgesia is an important clinical indicator in determining the quality of the care offered for emergency patients ${ }^{19}$. This time varies depending on the condition that caused the pain, but it should ideally be not more than $20-25 \mathrm{~min}^{20}$. In a recently published systematic review, it has been observed that the VAS scores at minute 30 post-procedure are considered in many studies on NSAIDs or opioids focusing on pain management in acute renal colic in the literature ${ }^{21}$. However, in the present study, the VAS scores not only at minute 30 but also at other times throughout the procedure were significantly lower in the ESP group. It was significant importance that the patients expressed their pain diminished immediately after the ESP block procedure. The pain scores at minute 5 already demonstrate that the pain reduced by $70 \%$ compared to the admittance pain scores.

There are still some considerations that need clarification although the NSAIDs are the first choice analgesics for acute renal colic due to their efficacy and levels of adverse effects. Renal dysfunction, gastrointestinal bleeding, and thrombosis dysfunction are the well-known adverse effects of NSAIDs. There are also differences that have been reported in terms of need for additional analgesic and adverse effects based on administration methods. Compared to opioids, NSAIDs have been demonstrated to have less need for additional analgesic when administered intravenously and less rates of vomiting when administered only intramuscularly ${ }^{22-24}$. On the other hand, despite the existing complications described for ESP block in the literature, there were not any complications or adverse effects during the course of the present study.

Since it is applied in various procedures such as FAST procedure in emergency departments, there is an increased familiarity with ultrasonography, and it sheds light on other procedures that can be applied under the guidance of ultrasonography. Ultrasound-guided application of regional anesthetic techniques at emergency departments gains popularity in pain management. Opioid consumption is getting less popular especially as a highly efficient, easily-applicable and safe component of multi-model analgesic. ESP block has been successfully employed at emergency departments in pain management of various clinical situations such as rib fractures, burns, herpes zoster, acute pancreatitis ${ }^{9}$. With the ESP block, the local anesthetic solution crosses the superior costotransverse ligament and spreads into the paravertebral space. Blockade of the ventral rami of the spinal nerves is the primary mechanism of the analgesic effect ${ }^{25}$. The most important reason of this common use of ESP block is because it is a highly safe plane block. Especially the vertebrae transverse process, which is the target point of the injection during the block, constitutes a safety point for the practitioner. This is therefore highly advantageous in outpatient procedures.

The present study has some limitations. The first of these limitations is although an ESP block takes relatively more time than intravenous analgesia with NSAIDs, in our institution, we perform ultrasoundguided ESP block in less than 5 min. Additional time, staff, and equipment may create a limitation in terms of time and cost-effectiveness. Therefore, further studies may be required to determine the cost-effectiveness of the study. The second limitation is that patient pain scores were followed up only for 60 min in the ED. Moreover the long-term outcomes of patients (sleep, physical function, depression, pain catastrophizing, etc.) were not evaluated according to our study protocol. If long-term results were evaluated, different results could have been obtained. Finally, possible biases could not have been prevented since the present study was designed as a study with no blindness for patients and practitioners.

\section{CONCLUSIONS}

We believe that ESP block can be an alternative, efficient and safe method to reduce pain in the analgesic 
management of acute renal colic pain. It reduces need for parenteral opioid. We are also of the opinion that there is a need for further clinical studies on the optimal analgesic drugs or techniques to be used in the analgesic management of acute renal colic.

\section{Figure Legends}

Figure 1: A-Sonographic anatomy of erector spinae plane block, B-Basic illustration of erector spinae plane block.

Figure 2: CONSORT flow diagram of the study.

\section{References}

1. Fisang C, Anding R, Muller SC, Latz S, Laube N. Urolithiasis-an interdisciplinary diagnostic, therapeutic and secondary preventive challenge. Deutsches Arzteblatt international. 2015;112(6):83-91.

2. Turk C, Knoll T, Petrik A, Sarica K, Straub M, Seitz C, eds.Guidelines on Urolithiasis. European Association of Urology; 2018.

3. Mozafari J, Maleki Verki M, Motamed H, Sabouhi A, Tirandaz F. Comparing intranasal ketamine with intravenous fentanyl in reducing pain in patients with renal colic: A double-blind randomized clinical trial. $A m$ J Emerg Med. 2019.

4. Jalili M, Shirani F, Entezari P, Hedayatshodeh M, Baigi V, Mirfazaelian H. Desmopressin/indomethacin combination efficacy and safety in renal colic pain management: A randomized placebo controlled trial. $\mathrm{Am}$ J Emerg Med. 2019;37(6):1009-1012.

5. Maleki Verki M, Porozan S, Motamed H, Fahimi MA, Aryan A. Comparison the analgesic effect of magnesium sulphate and Ketorolac in the treatment of renal colic patients: Double-blind clinical trial study. $A m$ J Emerg Med. 2019;37(6):1033-1036.

6. Eken C, Durmaz D, Erol B. Successful treatment of a persistent renal colic with trigger point injection. Am J Emerg Med.2009;27(2):252.e253-254.

7. Maldonado-Avila M, Del Rosario-Santiago M, Rosas-Nava JE, et al. Treatment of reno-ureteral colic by twelfth intercostal nerve block with lidocaine versus intramuscular diclofenac. Int Urol Nephrol.2017;49(3):413-417.

8. Nikiforov S, Cronin AJ, Murray WB, Hall VE. Subcutaneous paravertebral block for renal colic. Anesthesiology.2001;94(3):531-532.

9. Abdelhamid K, ElHawary H, Turner JP. The Use of the Erector Spinae Plane Block to Decrease Pain and Opioid Consumption in the Emergency Department: A Literature Review. J Emerg Med. 2020.

10. Forero M, Adhikary SD, Lopez H, Tsui C, Chin KJ. The Erector Spinae Plane Block: A Novel Analgesic Technique in Thoracic Neuropathic Pain.Regional anesthesia and pain medicine. 2016;41(5):621-627.

11. Ahiskalioglu A, Tulgar S, Celik M, Ozer Z, Alici HA, Aydin ME. Lumbar Erector Spinae Plane Block as a Main Anesthetic Method for Hip Surgery in High Risk Elderly Patients: Initial Experience with a Magnetic Resonance Imaging. The Eurasian journal of medicine.2020;52(1):16-20.

12. Kilicaslan A, Aydin A, Kekec AF, Ahiskalioglu A. Sacral erector spinae plane block provides effective postoperative analgesia for pelvic and sacral fracture surgery. Journal of clinical anesthesia.2020;61:109674.

13. Tekin E, Ahiskalioglu A, Aydin ME, Sengun E, Bayramoglu A, Alici HA. High-thoracic ultrasoundguided erector spinae plane block for acute herpes zoster pain management in emergency department. $A m$ J Emerg Med. 2019;37(2):375.e371-375.e373.

14. Aydin T, Balaban O, Ahiskalioglu A, Alici HA, Acar A. Ultrasound-guided Erector Spinae Plane Block for the Management of Herpes Zoster Pain: Observational Study. Cureus.2019;11(10):e5891. 
15. Aydin ME, Ahiskalioglu A, Tekin E, Ozkaya F, Ahiskalioglu EO, Bayramoglu A. Relief of refractory renal colic in emergency department: A novel indication for ultrasound guided erector spinae plane block. $A m$ J Emerg Med. 2019;37(4):794.e791-794.e793.

16. Thabane L, Ma J, Chu R, et al. A tutorial on pilot studies: the what, why and how. BMC Med Res Methodol. 2010;10:1.

17. Travaglini F, Bartoletti R, Gacci M, Rizzo M. Pathophysiology of reno-ureteral colic. Urol Int. 2004;72 Suppl 1:20-23.

18. McDougal WS, Wein AJ, Kavoussi LR, Partin AW, Peters CA. Campbell-Walsh Urology11th Edition. In: Elsevier Health Sciences; 2015.

19. Kelly AM, Brumby C, Barnes C. Nurse-initiated, titrated intravenous opioid analgesia reduces time to analgesia for selected painful conditions. Cjem. 2005;7(3):149-154.

20. HachimiIdrissi S, Coffey F, Dobias V, et al. Guidelines for the management of acute pain in emergency situations. : The European Society for Emergency Medicine;2020.

21. Pathan SA, Mitra B, Cameron PA. A Systematic Review and Meta-analysis Comparing the Efficacy of Nonsteroidal Anti-inflammatory Drugs, Opioids, and Paracetamol in the Treatment of Acute Renal Colic.Eur Urol. 2018;73(4):583-595.

22. Shirazi M, Salehipour M, Afrasiabi MA, Aminsharifi A. Analgesic Effects and Safety of Desmopressin, Tramadol and Indomethacin in Patients with Acute Renal Colic; A Randomized Clinical Trial.Bulletin of emergency and trauma. 2015;3(2):41-45.

23. Ay MO, Sebe A, Kozaci N, et al. Comparison of the analgesic efficacy of dexketoprofen trometamol and meperidine $\mathrm{HCl}$ in the relief of renal colic. American journal of therapeutics. 2014;21(4):296-303.

24. Zamanian F, Jalili M, Moradi-Lakeh M, Kia M, Aghili R, Aghili SM. Morphine Suppository versus Indomethacin Suppository in the Management of Renal Colic: Randomized Clinical Trial. Pain research and treatment. 2016;2016:4981585.

25. Adhikary SD, Bernard S, Lopez H, Chin KJ. Erector Spinae Plane Block Versus Retrolaminar Block: A Magnetic Resonance Imaging and Anatomical Study. Regional anesthesia and pain medicine. 2018;43(7):756762.
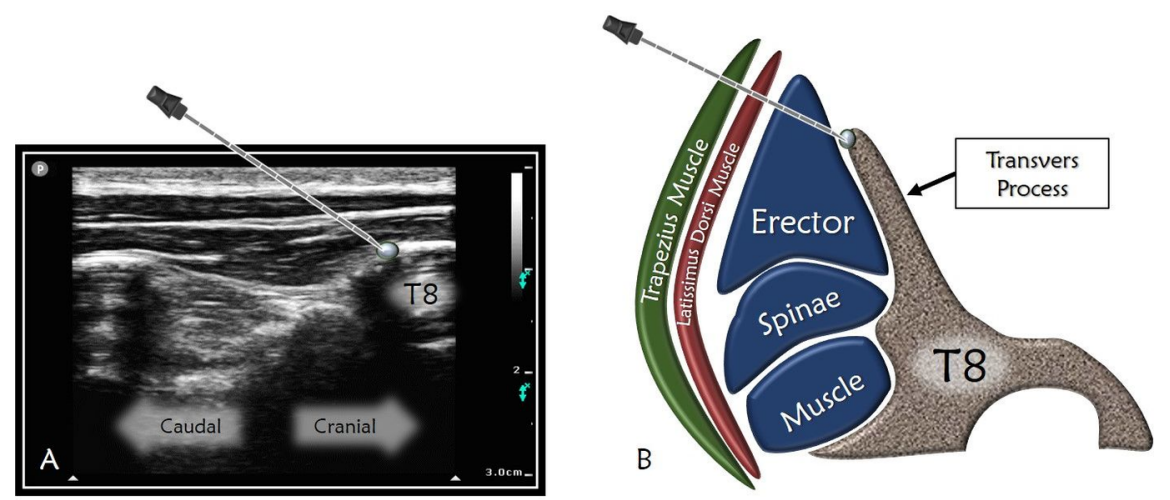

\section{Hosted file}

Figure 2.doc available at https://authorea.com/users/350483/articles/475311-erector-spinaeplane-block-vs-nonsteroidal-anti-inflammatory-drugs-for-severe-renal-colic-pain-a-pilotclinical-feasibility-study 


\section{Hosted file}

Tables.docx available at https://authorea.com/users/350483/articles/475311-erector-spinaeplane-block-vs-nonsteroidal-anti-inflammatory-drugs-for-severe-renal-colic-pain-a-pilotclinical-feasibility-study 